\title{
Probabilistic Analysis of Carlitz Compositions
}

\author{
Guy Louchard $^{1}$ and Helmut Prodinger ${ }^{2}$ \\ ${ }^{1}$ Université Libre de Bruxelles, Département d'Informatique, CP 212, Boulevard du Triomphe, B-1050 Bruxelles, \\ Belgium, \\ e-mail: louchardeulb.ac.be \\ ${ }^{2}$ University of the Witwatersrand, School of Mathematics, P.O. Wits, 2050 Johannesburg, South Africa, \\ e-mail: helmutestaff.ms.wits.ac.za
}

received Dec 31, 1999, accepted Apr 23, 2002.

\begin{abstract}
Using generating functions and limit theorems, we obtain a stochastic description of Carlitz compositions of large integer $n$ (i.e. compositions two successive parts of which are different). We analyze: the number $M$ of parts, the number of compositions $T(m, n)$ with $m$ parts, the distribution of the last part size, the correlation between two successive parts, leading to a Markov chain. We describe also the associated processes and the limiting trajectories, the width and thickness of a composition. We finally present a typical simulation. The limiting processes are characterized by Brownian Motion and some discrete distributions.
\end{abstract}

Keywords: Carlitz compositions, Smirnov words, polyominoes, central limit theorems, local limit theorems, large deviation theorems, Markov chains, Brownian motion

\section{Introduction}

A Carlitz composition of $n$ is a composition two successive parts of which are different (see Carlitz [6]). $T(m, n)$ will denote the number of Carlitz compositions of $n$ with $m$ parts. All $T(\cdot, n)$ compositions will be considered as equiprobable. We call $M$ the random variable (R.V.): number of parts. In [18], Knopfmacher and Prodinger found asymptotic values for: $T(\cdot, n)$, the mean number of parts $\mathbb{E}[M]$, the number of general compositions with exactly $m$ equal adjacent parts, the mean of equal adjacent parts number, the mean of the largest part. They also analyze Carlitz composition with zeros and with some restrictions. Carlitz compositions can also be studied using Smirnov words; see [110] and [18].

In [19], [20], and [21], Louchard analyzes some polyominoes (or animals) such as: directed columnconvex animals, column-convex animals and directed diagonally convex animals. He obtained asymptotic distributions for $M$ and for the size of columns. He also derived limiting processes for typical trajectories and shapes. Related material can be found in [3].

In the present paper, we consider Carlitz compositions as particular polyominoes and obtain a stochastic description of their behaviour for large $n$. We analyze the number $M$ of parts, the number of composition $T(m, n)$ with $m$ parts, the distribution of the last part size, the correlation between two successive parts, leading to a Markov chain. We consider also the associated processes and the limiting trajectories and 
shapes (which show a "filament silhouette"), the width and the thickness (part size) of a composition. We present an algorithm for efficient large Carlitz composition simulation.

We use such tools as asymptotic analysis of generating functions (based on their singularities) leading to central limit, local limit and large deviation theorems. The limiting processes are characterized by Brownian Motion, the extreme value-distribution and some discrete distributions.

Our results can be summarized as follows: the number $M$ of parts and $T(m, n)$ are analyzed in Theorems 2.1, 2.2, 2.3. The distribution of the last part size is given in Theorem 2.4. The distribution of intermediate part size and the correlation between two successive parts are considered in Theorems 2.5, 2.6 (including the Markov chain description). The associated processes are discussed in Theorems 2.7, 2.8, 2.9. The simulation algorithm is given in Sec. 3.1 and the thickness is considered in Theorem 3.1, 3.2, and 3.3.

The paper is organized as follows: Section 2 gives the probabilistic analysis of Carlitz compositions, Section 3 analyzes the simulation algorithm and the composition thickness, Section 4 concludes the paper. An Appendix provides some identities we need in the paper. Several notations will appear in the sequel. Let us summarize some of them:

- "D्D" means "convergence in distribution."

- $\mathcal{N}(\mu, V)$ is the Normal (or Gaussian) random variable with mean $\mu$ and variance $V$.

- “ " means "asymptotic to" ( $n$ or $m \rightarrow \infty)$.

- $T(m, n)$ is the number of Carlitz compositions of $n$ with $m$ parts.

- "Vertical distribution" means "Expression for $T(m, n)$ for given number of parts $m . "$

- "Horizontal distribution" means "distribution of the number of parts $M$ for given $n . "$

- $\Rightarrow_{n \rightarrow \infty}$ : weak convergence of random functions in the space of all right continuous functions having left limits with values in $R^{2}$ and endowed with the Skorohod metric $d_{0}$ (see Billingsley [4] Chap. III).

- $\operatorname{VAR}(\mathcal{T}):=\operatorname{Variance}(\mathcal{T})$.

- $B(t):=$ the classical Brownian Motion.

\section{Probabilistic analysis of Carlitz Compositions}

In this section, we first analyze the number $M$ of parts. Then we obtain the distribution of the last part size (LP). Next we compute the correlation between two successive intermediate parts, leading to a Markov chain. Finally, we consider the associated processes and limiting trajectories.

\subsection{Number of parts}

Let $T(m, n)$ be the number of Carlitz Compositions (CC) of $n$ with $m$ parts and let $f_{m}(i, n)$ be the number of $\mathrm{CC}$ with the same characteristics and last part of size $i$. We shall mark $n$ by $z, m$ by $w$ and $i$ by $\theta$. The corresponding generating functions (GF) satisfy $f_{j}(\theta, z)=f_{j-1}(1, z) \frac{z \theta}{1-z \theta}-f_{j-1}(z \theta, z), j \geq 2$ and $f_{1}=\frac{z \theta}{1-z \theta}$. (The first part can be of any size.) Hence $\phi(w, \theta, z):=\sum_{1}^{\infty} w^{j} f_{j}(\theta, z)$ satisfies

$$
\phi(w, \theta, z)=\frac{w z \theta}{1-z \theta}+\frac{w z \theta}{1-z \theta} \phi(w, 1, z)-w \phi(w, z \theta, z) .
$$




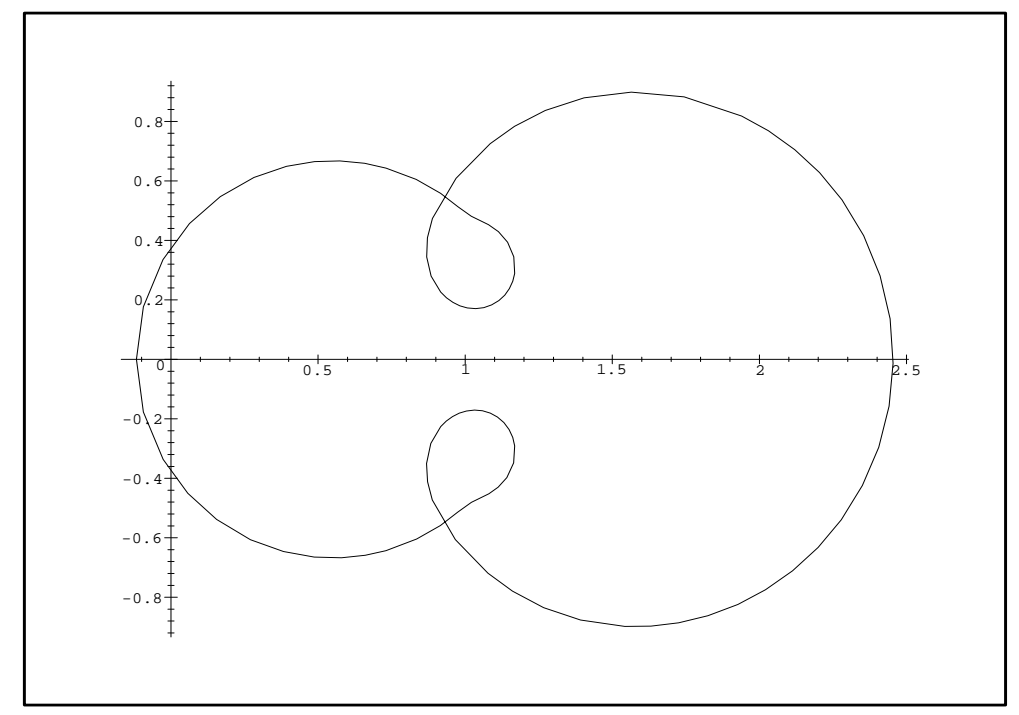

Fig. 1: Winding number of $h$

Iterating, this leads to

$$
\phi=A_{1}(w, \theta, z)\left[1+D_{1}\right]
$$

where

$$
\begin{aligned}
D_{1}:=\phi(w, 1, z) & =A_{1}(w, 1, z) /\left[1-A_{1}(w, 1, z)\right] \\
A_{1}(w, \theta, z) & =\sum_{j=1}^{\infty}(-1)^{j+1} \frac{z^{j} \theta w^{j}}{1-z^{j} \theta} .
\end{aligned}
$$

Notice that

$$
1+D_{1}=1 / h
$$

where $h(w, z):=1-A_{1}(w, 1, z)$. This generating function was already known to Carlitz [6].

First $\operatorname{set} \theta=1$. When $w=1$ we get the GF of the total number $T(\cdot, n)$ of $\mathrm{CC}(n)(\mathrm{CC}$ of $n)$ : this is given by $D_{1}(1, z)$. The dominant singularity of $D_{1}$ is given by the root $z^{*}$ (with smallest module) of $h(1, z)$, i.e. $z^{*}=.571349793158088 \ldots h(1, z)$ is analytic for $|z|<1$. To be sure that $z^{*}$ is the dominant singularity, we use the principle of the argument of Henrici [i1]: the number of solutions of the equation $f(z)=0$ that lie inside a simple closed curve $\Gamma$, with $f(z)$ analytic inside and on $\Gamma$, is equal to the variation of the argument of $f(z)$ along $\Gamma$, a quantity also equal to the winding number of the transformed curve $f(\Gamma)$ around the origin. The argument was used in Flajolet and Prodinger [8] in a similar situation. Figure [1] represents $[\Re(h), \mathfrak{I}(h)]$ for $z=.60 \exp (i t), t=0 . .2 \pi$, where $h$ is converted into a series up to $O\left(z^{60}\right)$. The winding number is 1 , so that $h$ has only one root $z^{*}$ for $|z|<.60$. 
By singularity analysis, $T(\cdot, n)$ is asymptotically given by

$$
T(\cdot, n) \sim-\frac{1}{z^{*} h_{z}\left(1, z^{*}\right)} \frac{1}{z^{* n}}=C_{1} / z^{* n}, \quad n \rightarrow \infty,
$$

with $C_{1}=\frac{1}{z^{*} A_{1, z}\left(1,1, z^{*}\right)}=\cdot 4563634741 \cdots$

$\left(F_{z}\right.$ means differentiation w.r.t. $z$ and similarly for $\left.w\right)$. Indeed $A_{1}\left(1,1, z^{*}\right) \equiv 1$. For $w \in[0,1], h$ is analytic for $|z|<1$ and for $w \in[1, \infty], h$ is analytic for $|z|<1 / w$. Let us remark that the numerical computation of $h$ is delicate: this is an alternating series. So it is better to use (A1). This leads to

$$
h(w, z)=1-\sum_{k \geq 1} \frac{w z^{k}}{1+w z^{k}} .
$$

The same technique has been used for $A_{1}(w, \theta, z)$ and $h_{w}, h_{z}$.

Let us return to our asymptotic analysis. By Bender's Theorems 1,3 and (3.2) in [2] we can get more: we obtain the asymptotic distribution of the number of parts $M$ in $\mathrm{CC}(n)$, where we consider all possible $T(\cdot, n)$ as equiprobable. Let

$$
\begin{aligned}
& r_{1}:=-h_{w} / h_{z} \\
& r_{2}:=-\left(r_{1}^{2} h_{z z}+2 r_{1} h_{z w}+h_{w}+h_{w w}\right) / h_{z} .
\end{aligned}
$$

Setting $w=1, z=z^{*}$, we derive

$$
\begin{aligned}
& \mu_{1}:=-r_{1} / z^{*}=\cdot 3506012746 \cdots, \\
& \sigma_{1}^{2}:=\mu_{1}^{2}-r_{2} / z^{*}=\cdot 1339166786 \cdots
\end{aligned}
$$

Then Bender's results lead to the following theorem.

Theorem 2.1 (Horizontal Distribution in $C C(n)$ ). The number $M$ of parts in a $C C(n)$ of large given $n$ is asymptotically Gaussian:

$$
\frac{M-n \mu_{1}}{\sqrt{n} \sigma_{1}} \stackrel{\mathcal{D}}{\sim} \mathcal{N}(0,1), \quad n \rightarrow \infty
$$

Also a local limit theorem holds:

$$
T(m, n) \sim \frac{C_{1}}{z^{* n}} \frac{e^{-\left(m-n \mu_{1}\right)^{2} /\left(2 n \sigma_{1}^{2}\right)}}{\sqrt{2 \pi n} \sigma_{1}}, \quad n \rightarrow \infty, m-n \mu_{1}=O(\sqrt{n}) .
$$

Remark. $C_{1}$ and $\mu_{1}$ are already given in Knopfmacher and Prodinger [118].

For $n=30$, Figure 2 gives the observed normalized distribution of $M$ (observed = circle, asymptotic = line), extracted from $\left[z^{30}\right] D_{1}$. There is an obvious bias: this will be explained later in Section 2.2.

Now if we fix $m$ and consider $n$ as variable (there are, of course, an infinite number of CC for given $m$ ), we can obtain another asymptotic form for $T(m, n)$ : the conditioned distribution is given by

$$
\left[w^{m} z^{n}\right] D_{1}(w, z)=\frac{1}{z^{* n}}\left[w^{m} z^{n}\right] D_{1}\left(w, z z^{*}\right) .
$$




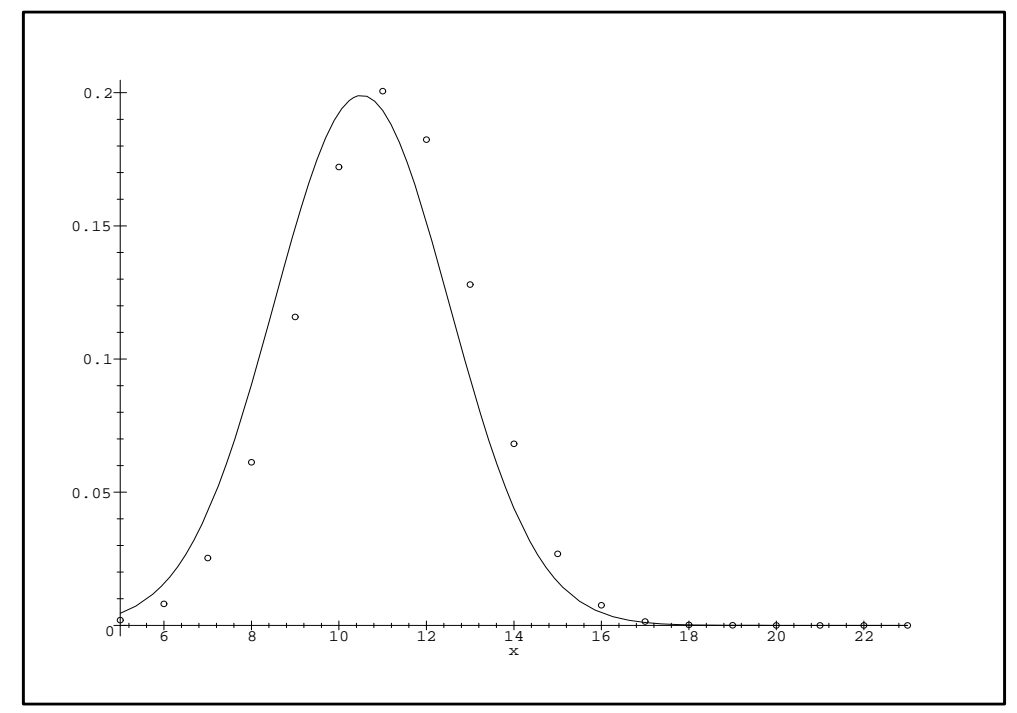

Fig. 2: Observed normalized distribution of $M(n=30)$

But, for $z=1$, the dominant singularity of $D_{1}\left(w, z z^{*}\right)$ is $w^{*}=1$. So with

$$
\begin{aligned}
C_{2} & =\frac{-1}{h_{w}\left(1, z^{*}\right)}=1 \cdot 3016594836 \cdots, \\
\mu_{2} & :=1 / \mu_{1}=2 \cdot 852242911 \cdots, \\
\sigma_{2}^{2} & =\sigma_{1}^{2} / \mu_{1}^{3}=3 \cdot 1073787943 \cdots,
\end{aligned}
$$

we obtain the following theorem.

Theorem 2.2 (Vertical Distribution in $C C(n)$ ). For large given number of parts $m, T(m, n)$ is asymptotically given by

$$
T(m, n) \sim \frac{C_{2}}{z^{* n}} \frac{e^{-\left(n-m \mu_{2}\right)^{2} /\left(2 m \sigma_{2}^{2}\right)}}{\sqrt{2 \pi m} \sigma_{2}}, \quad m \rightarrow \infty, n-m \mu_{2}=O(\sqrt{m}) .
$$

Note that $C_{1} / C_{2}=\mu_{1}$.

For further use, we have also computed $C_{2}(j)$ based on a first part of fixed size $j$. We first derive (conditioning on $j$ ):

$$
\phi(w, \theta, z \mid j)=A_{2}(w, \theta, z \mid j)+A_{1}(w, \theta, z) D_{2},
$$

where

$$
\begin{aligned}
D_{2} & :=\phi(w, 1, z \mid j)=A_{2}(w, 1, z \mid j) / h, \\
A_{2}(w, \theta, z \mid j) & =\theta^{j} w z^{j} /\left(1+w z^{j}\right),
\end{aligned}
$$


and $A_{1}$ is given by (2).

This leads to

$$
C_{2}(j)=-\frac{A_{2}\left(1,1, z^{*} \mid j\right)}{h_{w}\left(1, z^{*}\right)}=\frac{-z^{* j}}{1+z^{* j}} \frac{1}{h_{w}\left(1, z^{*}\right)} .
$$

Note that, by (A1), $\sum_{j} C_{2}(j)=C_{2}$, as it should. Theorem 2.3 is still valid.

Bender's theorems are based on the analysis of $\varphi(w)=\left[\frac{r(1)}{r(w)}\right]^{n}$ where $r(w)$ is the root of (3), seen as a $z$-equation, and $r(1)=z^{*}$. But $r(1) / r(w)$ is usually not a Probability GF (PGF). Indeed, we obtain $\frac{1}{r(w)}=1+w-\frac{1}{2} w^{2}+\frac{7}{12} w^{3}+\cdots$ which of course doesn't correspond to a PGF. Similarly, we could consider $t(z)$, the root of (3) (seen as a $w$-equation), with $t\left(z^{*}\right)=1$, and analyze $\frac{1}{t\left(z^{*} z\right)}$. But we can't even construct a series in $z$.

It is possible to derive a large deviation result for the number of parts, using $r(w)$.

Following Bender [2] Theorem 3, we first set $w=e^{s}$. We have

$$
\begin{aligned}
r^{\prime}(s) & =-h_{w} e^{s} / h_{z}, \\
r^{\prime \prime}(s) & =-\left(r^{\prime 2} h_{z z}+2 r^{\prime} e^{s} h_{z w}+e^{s} h_{w}+e^{2 s} h_{w w}\right) / h_{z} .
\end{aligned}
$$

where, here, $h_{w}=h_{w}(w, r(w))$, etc.

Bender leads to the following large deviation result:

Theorem 2.3 For $m$ outside the range $\left[n \mu_{1}-O(\sqrt{n}), n \mu_{1}+O(\sqrt{n})\right]$, choose $s^{*}$ such that $\mu=m / n=$ $-r^{\prime}\left(s^{*}\right) / r\left(s^{*}\right)$. The following asymptotic relation holds:

$$
T(m, n) \sim \frac{e^{-m s^{*}} A_{1}\left[e^{s^{*}}, 1, r\left(s^{*}\right)\right]}{\left[r\left(s^{*}\right)\right]^{n} \sigma\left(s^{*}\right) \sqrt{2 \pi n}\left[-h_{z}\left(e^{s^{*}}, r\left(s^{*}\right)\right)\right] r\left(s^{*}\right)}, \quad n \rightarrow \infty,
$$

with $\sigma^{2}\left(s^{*}\right)=(m / n)^{2}-r^{\prime \prime}\left(s^{*}\right) / r\left(s^{*}\right)$.

The range of validity of Theorem 2.3 can be established as follows. First, we must consider the contour of $h(w, z)=0$. After detailed analysis, it appears that this contour $h(w, z)=0$ is made of 2 parts: see Figure 3 .

Part I, for $w \in(0,1], z \in\left[z^{*}, 1\right)$, with $r(w) \underset{w \rightarrow 0}{\longrightarrow} 1$.

Part II, for $w \in\left[1, w_{1}\right), z \in\left(1 / w_{1}, z^{*}\right]$ and $w_{1}$ is the limit of the solution of $h\left[w, \frac{1}{w}-\varepsilon\right]=0, \varepsilon \rightarrow 0$.

The computation of $w_{1}$ offers no special numerical problem and leads to

$$
w_{1}=2 \cdot 584243690 \cdots, z_{1}=1 / w_{1}=\cdot 3869604109 \cdots
$$

(we must only be careful with the precision: 20 digits are enough in the range we use). $\mu\left(w_{1}\right)$ and $\sigma^{2}\left(w_{1}\right)$ are given by

$$
\mu\left(w_{1}\right)=\cdot 4657044011 \cdots, \sigma^{2}\left(w_{1}\right)=\cdot 1056119001 \cdots .
$$

The function $\mu(w)$ is increasing from 0 to $\mu\left(w_{1}\right), \sigma^{2}(w)$ goes from 0 (for $w=0$ ) to some maximum and then decreases to $\sigma^{2}\left(w_{1}\right)$. So finally, the range for $\mu$ is given by $\left(0, \mu\left(w_{1}\right)\right), z=r(w)$ following the contour defined above.

The verification of condition (V) of Bender's Theorem 3 (which allows to go from a central limit theorem to a local limit theorem) amounts to prove that $h\left(e^{s}, z\right)$ is analytic and bounded for

$$
|z| \leq|r(\Re(s))|(1+\delta) \quad \text { and } \quad \varepsilon \leq|\mathfrak{I}(s)| \leq \pi,
$$




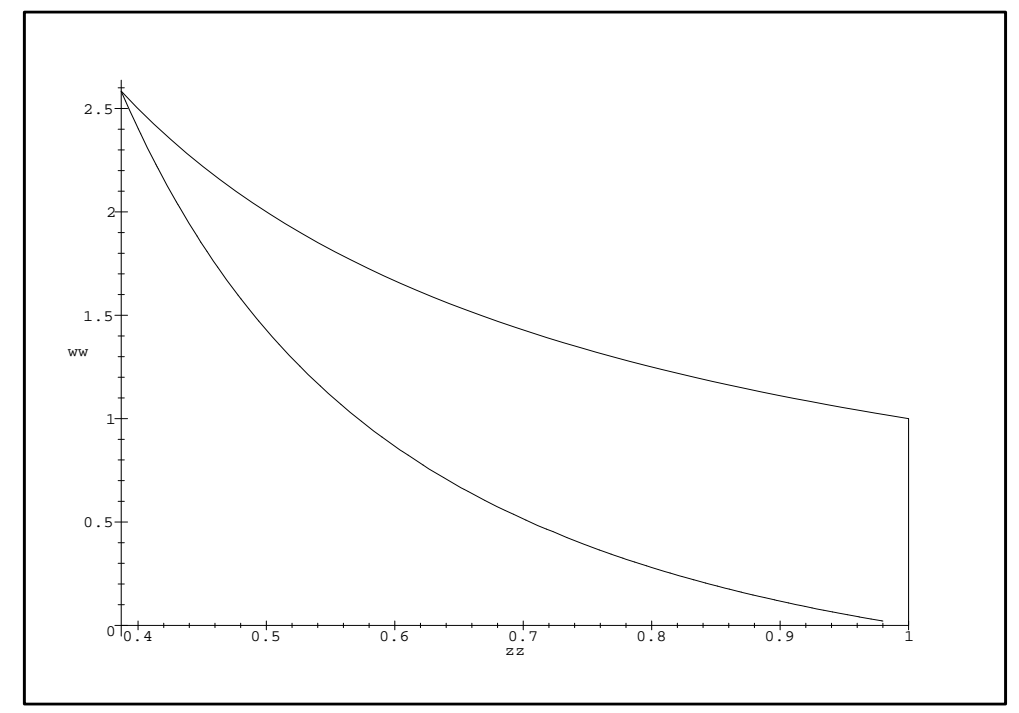

Fig. 3: Contour of $h=0$ and domain of convergence

for some $\varepsilon>0, \delta>0$, where $r(s)$ is the suitable solution of $h\left(e^{s}, r(s)\right)=0$ (i.e. with $\left.r(0)=z^{*}\right)$ : this follows from the analyticity analysis of $h$ in the beginning of this section.

\subsection{Distribution of the last part size in CC}

We want to analyze the asymptotic distribution of the size LP of the last part of a CC $(n)$. Setting $w=1$ in (II), we derive

$$
\left[z^{n}\right] \phi(1, \theta, z) \sim \frac{A_{1}\left(1, \theta, z^{*}\right)}{-z^{* n} z^{*} h_{z}\left(1, z^{*}\right)}, \quad n \rightarrow \infty,
$$

uniformly for $\theta$ in some complex neighbourhood of the origin. This may be checked by the method of singularity analysis of Flajolet and Odlyzko, as used in Flajolet and Soria [9] or Hwang [13]. Normalizing by the total number of $\mathrm{CC}(n)$ (4) this leads to the following asymptotic $(n \rightarrow \infty)$ PGF for the last part size LP:

$$
G(\theta)=A_{1}\left(1, \theta, z^{*}\right)
$$

Expanding, this leads to

$$
\pi_{1}(i):=\left[\theta^{i}\right] G(\theta)=\frac{z^{* i}}{1+z^{* i}}
$$

with typical geometric behaviour. Of course $G(1)=1$ by (A1) and $E(L P)=\sum i \pi(i)=2 \cdot 5833049669 \cdots$.

For $n=12$, we have computed the observed normalized distribution of $L P$ corresponding to

$$
\left[z^{12}\right] \phi(\theta, 1, z)
$$




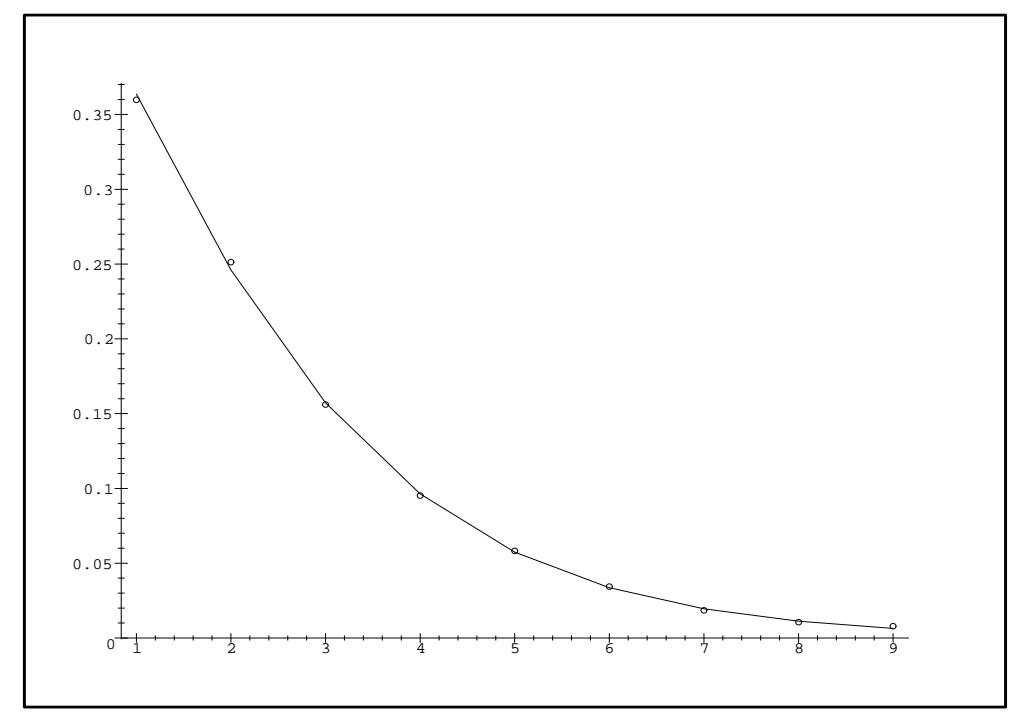

Fig. 4: Observed normalized distribution of the last part size $\operatorname{LP}(n=12)$.

This gives the following Figure 4 (observed $=$ circle, asymptotic $=$ line). The agreement is quite good.

We can now obtain more information from (II). The asymptotic distribution of the last part size in $\mathrm{CC}(n)$ of $m$ parts is related to $\left[w^{m} z^{n}\right] \phi(w, \theta, z)$ and Bender's Theorems 1 and 3 lead to the GF:

$$
T(\theta, m, n) \sim \frac{C_{1}}{z^{* n}} \frac{e^{-\left(m-n \mu_{1}\right)^{2} /\left(2 n \sigma_{1}^{2}\right)}}{\sqrt{2 \pi n} \sigma_{1}} G(\theta),
$$

for $m-n \mu_{1}=O(\sqrt{n})$, uniformly for $\theta$ in some complex neighbourhood of the origin. Again the uniformity can be checked by following Bender's analysis. Normalizing by $T(m, n)$ (see Theorem 2.1), this gives again $G(\theta)$. So we have proved the following result:

Theorem 2.4 For $m-n \mu_{1}=O(\sqrt{n})$, the asymptotic distribution of the size LP of the last part in $C C(n)$ with $m$ parts is given by $\pi_{1}(j)=z^{* j} /\left(1+z^{* j}\right)$ for $n \rightarrow \infty$ uniformly for all $j$.

For instance, for $n=30, m=9,11,13,\left(\left\lfloor n \mu_{1}\right\rfloor=10,\left\lfloor\sqrt{n} \sigma_{1}\right\rfloor=2\right)$ we have computed the observed normalized distribution of LP.

Figure 5 shows that the fit is quite good near the mean, but there is a bias for the two other values of $m$ : $n$ is not yet large enough to yield a distribution of LP that is nearly independent of $m$

Returning to the proof of Bender's theorem, we must compute from (1) $\left[\theta^{k} w^{m}\right] \varphi_{1} \varphi_{2}\left[\frac{z^{*}}{r(w)}\right]^{n}$ with

$$
\begin{aligned}
\varphi_{1}(\theta, w) & =A_{1}(w, \theta, r(w)) \\
\varphi_{2}(w) & =-\frac{1}{r(w) h_{z}(w, r(w))},
\end{aligned}
$$




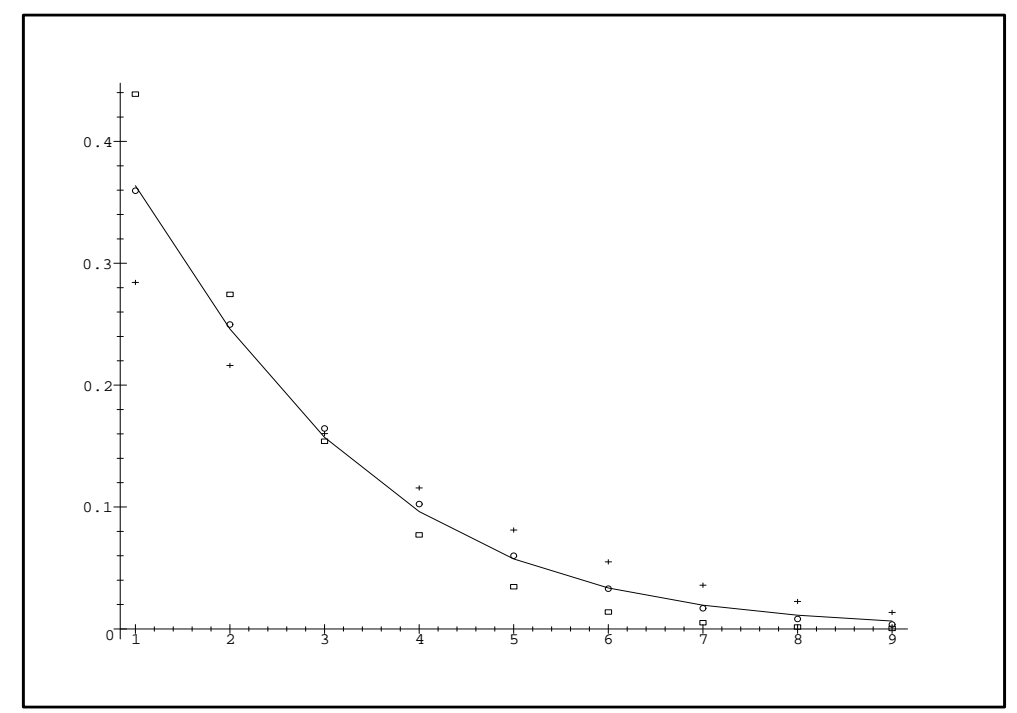

Fig. 5: Observed normalized LP distribution (cross: $m=9$, circle: $m=11$, box: $m=13, n=30$ )

and $r(w)$ is again the root of (3) with $r(1)=z^{*}$. But according to Hwang [13], [14, Theorem 2], we know that the mean value of $M$ for large $n$ is given by $\mathbb{E}(M) \sim n \mu_{1}+v^{\prime}(0)$, where

$$
v(s):=\log \left[\varphi_{1}\left(1, e^{s}\right) \varphi_{2}\left(e^{s}\right) /\left(\varphi_{1}(1,1) \varphi_{2}(1)\right)\right],
$$

i.e.

$$
v^{\prime}(0)=\frac{\varphi_{1, w}(1,1)}{\varphi_{1}(1,1)}+\frac{\varphi_{2, w}(1)}{\varphi_{2}(1)}=\beta \quad \text { say. }
$$

But it is easy to check that $\varphi_{1, w}(1,1)=0$ and

$$
\begin{aligned}
\varphi_{2, w}(1) & =\left[r_{w} h_{z}+z^{*} h_{z, z} r_{w}+z^{*} h_{z, w}\right] /\left[z^{*} h_{z}\right]^{2}, \\
\varphi_{2}(1) & =-1 /\left(z^{*} h_{z}\right) \\
r_{w} & =-h_{w} / h_{z} .
\end{aligned}
$$

From now on, we set $h_{z}=h_{z}\left(1, z^{*}\right)$, etc.

Numerical computation gives $\beta=.5365417012 \cdots$ This bias $\beta$ is now reintroduced in Figure $1(n=30)$. This gives Figure 6, where the fit is now nearly perfect.

\subsection{Correlation between two successive intermediate parts}

Let us now turn to two successive parts $m_{1}, m_{1}+1$, of size $k, j$, such that their distances from the first and the last part are of order $O(n)$. Let $T(m, n, j)$ be the total number of $\mathrm{CC}(n)$ with $m$ parts and last part size 


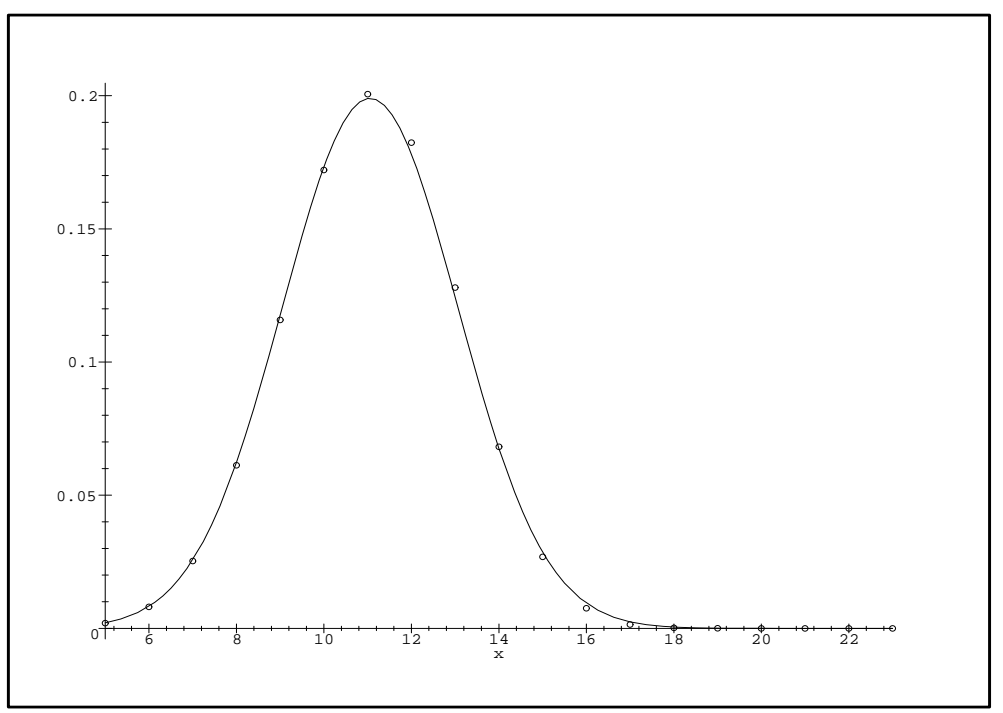

Fig. 6: Observed normalized distribution of $M$ with bias (observed $=$ circle, asymptotic $=$ line, $n=30$ )

$j$, let $\#\left(m, n, m_{1}, k\right)$ be the total number of $\mathrm{CC}(n)$ with $m$ parts, part $m_{1}$ of size $k$, and set $m_{2}:=m-m_{1}$. We have, conditioning on $j$,

$$
\#\left(m, n, m_{1}, k\right)=\sum_{n_{1}} \sum_{j \neq k} T\left(m_{1}, n_{1}, k\right) T\left(m_{2}, n-n_{1}, \cdot\right) \mid j .
$$

With Theorem 2.4 (vertical form) and Theorem 2.2 [Eq. 7], this leads, after normalization by $T(m, n)$, to the following distribution:

$$
\begin{aligned}
\frac{\#\left(m, n, m_{1}, k\right)}{T(m, n)} \sim & \sum_{n_{1}} \sum_{j \neq k} \frac{e^{-\left(n_{1}-m_{1} \mu_{2}\right)^{2} / 2 m_{1} \sigma_{2}^{2}}}{\sqrt{2 \pi m_{1}} \sigma_{2}} \pi_{1}(k) \times \\
& \times C_{2}(j) \frac{e^{-\left(n-n_{1}-m_{2} \mu_{2}\right)^{2} / 2 m_{2} \sigma_{2}^{2}}}{\sqrt{2 \pi m_{2}} \sigma_{2}} / \frac{e^{-\left(n-m \mu_{2}\right) / 2 m \sigma_{2}^{2}}}{\sqrt{2 \pi m} \sigma_{2}}
\end{aligned}
$$

and with (A1) we readily obtain the following distribution $\pi_{2}(k)$ for $k$ :

$$
\pi_{2}(k)=\sum_{j \neq k} \pi_{1}(k) C_{2}(j)=\frac{-z^{* k}}{\left(1+z^{* k}\right)^{2} h_{w}} .
$$

For further use, we compute, for $\ell \geq k$

$$
\pi_{2}(\ell)=\pi_{2}^{(1)}(\ell) z^{* k}+\pi_{2}^{(2)}(\ell) z^{* 2 k}+O\left(z^{* 3 k}\right), \quad k \rightarrow \infty
$$


with

$$
\pi_{2}^{(1)}(\ell)=-\frac{z^{*(l-k)}}{h_{w}}, \quad \pi_{2}^{(2)}(\ell)=\frac{2 z^{* 2(l-k)}}{h_{w}} .
$$

By (A2), we obtain $\sum_{1}^{\infty} \pi_{2}(k)=1$ and by (A3), $\sum k \pi_{2}(k)=\mu_{2}$ as it should. So we obtain the following theorem.

Theorem 2.5 The asymptotic $(n \rightarrow \infty)$ distribution of intermediate part size is given by $\pi_{2}(k)$, with mean $\mu_{2}$.

But we can get more from (8), which shows that the asymptotic joint distribution (in stationary distribution) of two successive intermediate part sizes $k$ and $j$ is given by

$$
\pi_{1}(k) C_{2}(j), j \neq k
$$

Normalizing by $\pi_{2}(k)$, this leads to the following Markov chain related to two successive intermediate parts:

$$
\Pi(k, j)=\frac{z^{* j}\left(1+z^{* k}\right)}{1+z^{* j}}, j \neq k .
$$

The chain is irreducible, recurrent positive and reversible. Of course the stationary distribution of $\Pi$ is given by $\pi_{2}(k)$. Therefore, we obtain the following theorem.

Theorem 2.6 The asymptotic, $n \rightarrow \infty$, distribution of two successive intermediate parts in a CC (n) is given by a Markov chain $\Pi(i, j)$, with stationary distribution $\pi_{2}(k)$, and with mean $\mu_{2}$.

Let us now analyze the asymptotic behaviour of $\Pi(i, j)$. This will be used in Sec. 3.2. For fixed $\ell$, we obtain

$$
\Pi^{+}(\ell, k):=\sum_{j=k}^{\infty} \Pi(\ell, j)=\left(1+z^{* \ell}\right) \frac{z^{* k}}{1-z^{*}}\left(1+O\left(z^{* k}\right)\right), \quad k \rightarrow \infty .
$$

For further use, we set

$$
\varphi_{3}(\ell):=\frac{1+z^{* \ell}}{1-z^{*}}
$$

\subsection{Associated processes and limiting trajectories}

Let us now turn to trajectories. Let $x_{i}$ be the size of part $i$ and set $X(j):=\sum_{1}^{j} x_{i}$. We have $E[X(m)] \sim m \mu_{2}$ and we must check that $\operatorname{VAR}[X(m)] \sim m \sigma_{2}^{2}$ as given by (5). We first derive $m \sigma_{X}^{2}:=\operatorname{VAR}[X(m)] \sim m\left[S_{2}-\right.$ $\left.\mu_{2}^{2}\right]+2 m \sum_{1}^{\infty} C_{k}^{x}$ where

$$
\begin{aligned}
S_{2} & :=\sum_{2} \pi_{2}(j) j^{2}=12 \cdot 208296194 \cdots, \\
C_{k}^{x} & :=\sum_{i} \sum_{j}\left(i-\mu_{2}\right) \pi_{2}(i) \Pi^{k}(i, j)\left(j-\mu_{2}\right) .
\end{aligned}
$$

But it is easily checked that $S_{3}:=\sum_{1}^{\infty} C_{k}^{x}$ can be written as

$$
S_{3}=\lim _{w \rightarrow 1}\left[-\frac{1}{h_{w}}\left\{\sum_{i} \sum_{j} \frac{i}{1+z^{* i}}\left[\theta^{j}\right] \phi\left(w, \theta, z^{*} \mid i\right) \frac{j}{1+z^{* j}}-w \sum_{i} \frac{z^{* i} i^{2}}{\left(1+z^{* i}\right)^{2}}\right\}-\mu_{2}^{2} \frac{w^{2}}{1-w}\right] .
$$


With (6), this can be simplified as

$$
\begin{aligned}
S_{3}= & \lim _{w \rightarrow 1}\left[\sum_{i} \sum_{j}-\frac{1}{h_{w}} \frac{i}{1+z^{* i}} \frac{j}{1+z^{* j}}\left[\theta^{j}\right]\left\{\frac{A_{2}\left(w, \theta, z^{*} \mid i\right)-w z^{* i} \theta^{i}}{w}\right.\right. \\
& \left.\left.+\frac{A_{1}\left(w, \theta, z^{*}\right) A_{2}\left(w, 1, z^{*} \mid i\right)}{w h\left(w, z^{*}\right)}\right\}-\frac{\mu_{2}^{2} w}{1-w}\right] .
\end{aligned}
$$

This leads to (we set $w=1-\varepsilon$ )

$$
S_{3}=\lim _{\varepsilon \rightarrow 0}\left[-\frac{\mu_{2}^{2}}{\varepsilon}+\mu_{2}^{2}+\bar{\varphi}_{6}-\frac{1}{h_{w}}\left[-\frac{\bar{\varphi}_{5}(1)}{h_{w} \varepsilon}+\frac{\bar{\varphi}_{5, w}(1)}{h_{w}}-\frac{\bar{\varphi}_{5}(1) h_{w w}}{2 h_{w}^{2}}\right]\right],
$$

where

$$
\begin{aligned}
\bar{\varphi}_{6} & :=\frac{1}{h_{w}} \sum_{i} \frac{i^{2}}{\left(1+z^{* i}\right)^{2}} \frac{z^{* 2 i}}{\left(1+z^{* i}\right)}=-.95938032262 \cdots, \\
\varphi_{5}(w, \theta, i) & =A_{1}\left(w, \theta, z^{*}\right) A_{2}\left(w, 1, z^{*} \mid i\right) / w .
\end{aligned}
$$

But $A_{1}\left(1, \theta, z^{*}\right) \equiv G(\theta)$, so we derive

$$
-\frac{1}{h_{w}} \cdot \frac{-\bar{\varphi}_{5}(1)}{h_{w}}=-\frac{1}{h_{w}} \sum_{i} \sum_{j}\left[\frac{i}{1+z^{* i}} \cdot \frac{-z^{* i}}{\left(1+z^{* i}\right) h_{w}} \cdot \frac{z^{* j}}{1+z^{* j}} \cdot \frac{j}{1+z^{* j}}\right] \equiv \mu_{2}^{2} .
$$

So the singularity in (12) is removed.

Similarly, we obtain

$$
\bar{\varphi}_{5, w}(1)=\sum_{i} \sum_{j}\left[\frac{i}{1+z^{* i}} \cdot \frac{j}{1+z^{* j}}\left[\theta^{j}\right] \varphi_{5, w}(1, \theta, i)\right]
$$

and finally

$$
m \sigma_{X}^{2} \sim m\left[S_{2}+\mu_{2}^{2}+2\left\{\bar{\varphi}_{6}-\frac{\bar{\varphi}_{5, w}(1)}{h_{w}^{2}}+\frac{\mu_{2}^{2} h_{w w}}{2 h_{w}}\right\}\right]=m \sigma_{2}^{2},
$$

by (A4) as expected.

We can now apply the Functional Central Limit Theorem (for dependent R.V.) (see for instance Billingsley [4, p. $168 \mathrm{ff}$.$] ) and we obtain the following result, where B(t)$ is the standard Brownian Motion (BM).

\section{Theorem 2.7}

$$
\frac{X([N t])-\mu_{2} N t}{\sigma_{2} \sqrt{N}} \Rightarrow B(t), \quad N \rightarrow \infty, \quad t \in[0,1]
$$

where $N:=$ number of parts of $\mathrm{CC}(n)$.

Let us now condition on $X(m)=n$. A realization of $X$ for fixed $n$ is given by Theorem 2.7, where we stop at a random time $m$ such that $X(m)=n$. Proceeding as in [19] it is easy to check that this amounts to fix $N=n / \mu_{2}$ in Theorem 2.7 (denominator) and we obtain the following result. 
Theorem 2.8 Conditioned on $X(m)=n$,

$$
\frac{X([m t])-\mu_{2} m t}{\sigma_{2} \sqrt{n / \mu_{2}}} \Rightarrow B(t)
$$

Let us now consider the oscillations of $X(\cdot)$ around its mean. Let us define the lower oscillation bound $W_{n}^{-}$and the range $W_{n}$ :

$$
\begin{aligned}
W_{n}^{-} & :=\underset{i \in[0, m]}{-\inf }\left[X(i)-\mu_{2} i\right], \\
W_{n} & :=\left\{\begin{array}{cc}
\sup _{[0, m]}- & \inf _{0, m]}
\end{array}\right\}\left[X(i)-\mu_{2} i\right],
\end{aligned}
$$

where $m$ is the number of parts in $\mathrm{CC}(n)$.

But now the lower bound $W_{n}^{-}$, normalized by $\sqrt{n / \mu_{2}} \sigma_{2}$, is asymptotically given by inf $B(t)$ and $[0,1]$

the range $W_{n}$ corresponds to the range $\left\{\begin{array}{ll}\sup _{[0,1]}- & \inf _{[0,1]}\end{array}\right\} B(t)$. The densities of these R.V. are well known: the first one is given by

the second one is given by

$$
f_{1}(x)=\frac{2 e^{-x^{2} / 2}}{\sqrt{2 \pi}}
$$

$$
f_{2}(x)=\frac{8}{\sqrt{2 \pi}} \sum_{k=1}^{\infty} k^{2}(-1)^{k-1} \exp \left[-k^{2} x^{2} / 2\right]
$$

or

where

$$
f_{2}(x)=\left(\frac{2}{\pi}\right)^{1 / 2} \frac{1}{x} L^{\prime}\left(\frac{x}{2}\right),
$$

$$
\begin{aligned}
L(z) & =1-2 \sum_{k=1}^{\infty}(-1)^{k-1} \exp \left[-2 k^{2} z^{2}\right] \\
& =(2 \pi)^{1 / 2} \frac{1}{z} \sum_{k=1}^{\infty} \exp \left[-2(k-1)^{2} \pi^{2} / 8 z^{2}\right],
\end{aligned}
$$

by Jacobi $\theta$-relations (see e.g. Whittaker and Watson [22].

This immediately leads to the following result

Theorem 2.9 For large $n$, and with $N=n / \mu_{2}$,

$W_{n}^{-} /\left(\sigma_{2} \sqrt{N}\right)$ has the asymptotic density $f_{1}$,

$W_{n} /\left(\sigma_{2} \sqrt{N}\right)$ has the asymptotic density $f_{2}$.

In summary, we can see the CC as a B.M. with some thickness (part size). The distribution of the thickness is characterized by Theorem 2.6. 


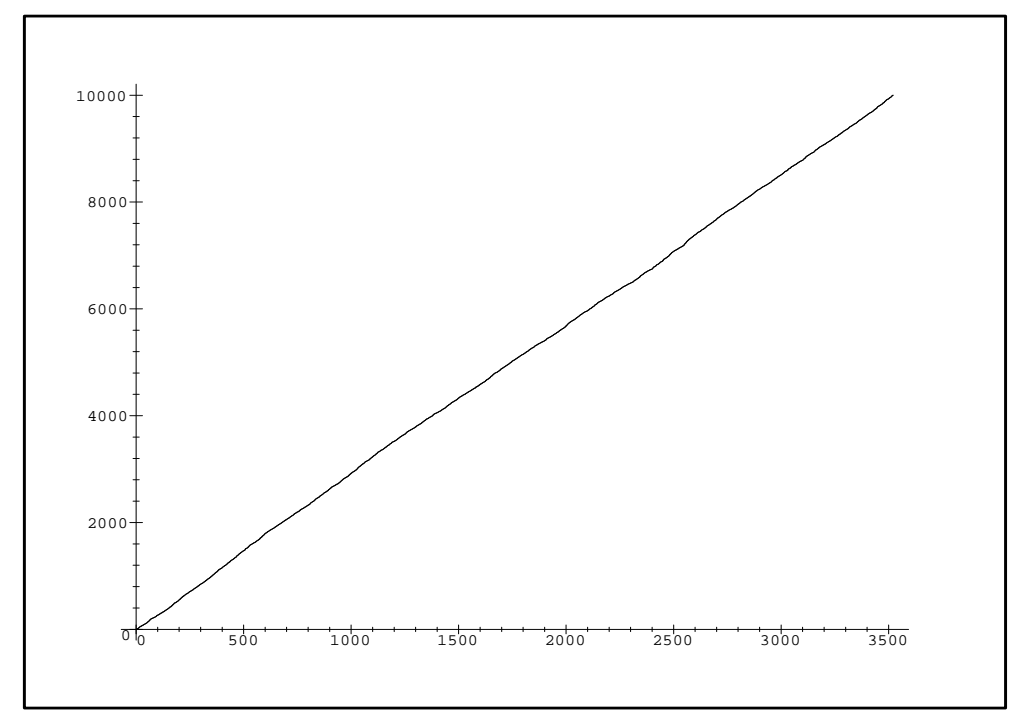

Fig. 7: Unnormalized $X(\cdot)$.

\section{Simulations and thickness}

In this section, we present an algorithm for $\mathrm{CC}(n)$ simulation, then we analyze the $\mathrm{CC}$ thickness maximum.

\subsection{Realizations}

A simulated realization of a $\mathrm{CC}(n)$ proceeds as follows. $\square$ Start with a part of size $j$ at time $i=1$ given by $\pi_{1}(j)$. Proceed from a part of size $k$ at time $i-1$ to a part of size $j$ at time $i$ by using a Markov chain defined by the probability matrix $\Pi(k, j)$ given by (10). Stop the chain as soon as $S:=\sum_{i=1}^{m} j_{i}>n$.

For $n=10.000$, a typical unnormalized trajectory for $X(i)$ is given in Figure 7, which shows a "filament silhouette." A typical normalized trajectory for $\frac{X(i)-\mu_{2} i}{\sigma_{2} \sqrt{N}}$ and $\frac{X(i-1)-\mu_{2} i}{\sigma_{2} \sqrt{N}}$, showing the thickness (here the thickness is defined by the size of the parts) is given in Figure 8. Of course, both trajectories are asymptotically equivalent for $n \rightarrow \infty$. A zoom on $i=[1030 . .1050]$ is given in Figure 9 .

\subsection{Hitting times and maximum for CC thickness}

Let us call thickness the part size. In this section, we derive the thickness hitting times (to high level) asymptotic distribution. This leads to a maximum asymptotic density. We consider the set $x_{i}$ of R.V. describing the thickness of CC. Let us define the set $D:=[k . . \infty), k>>1$. By (11), we see that the probability transition to $D$ is $O(\varepsilon), \varepsilon=z^{* k}$ and by standard properties (see Keilson [16], Aldous [1]]) we know that the hitting time to $D$ is such that (we drop $k$ for ease of notation):

- $E_{\ell}\left[T_{D}\right]=\frac{C_{3}}{\varepsilon}+\psi(\ell)+O(\varepsilon)$ (Actually a Laurent series exists for $\varepsilon$ sufficiently small), 


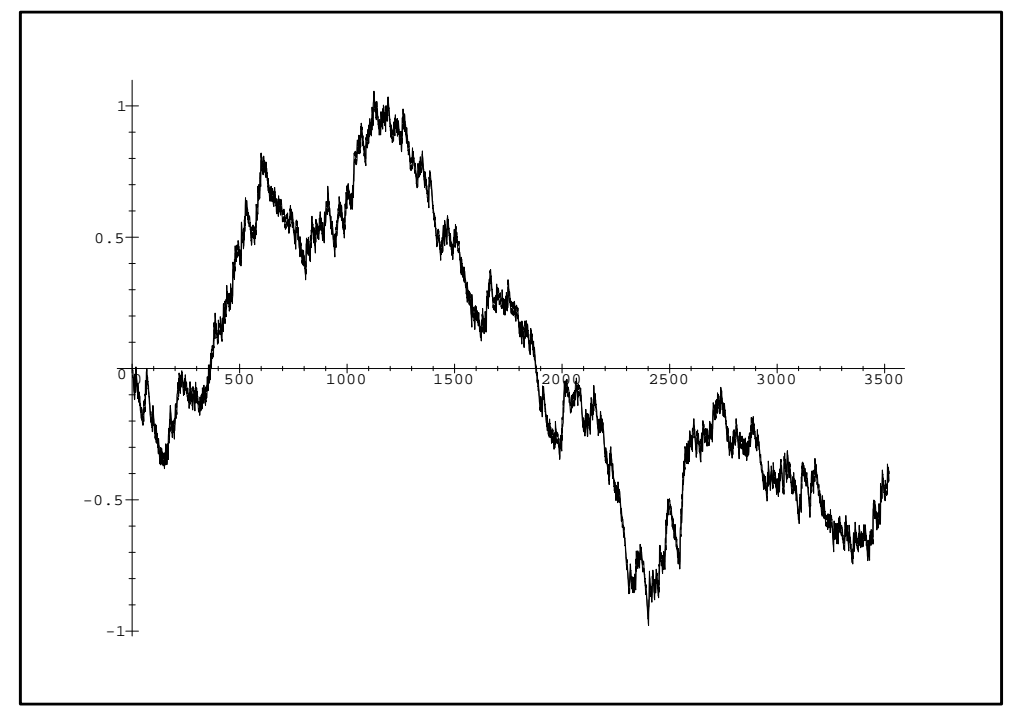

Fig. 8: Normalized $X(\cdot)$, with thickness.

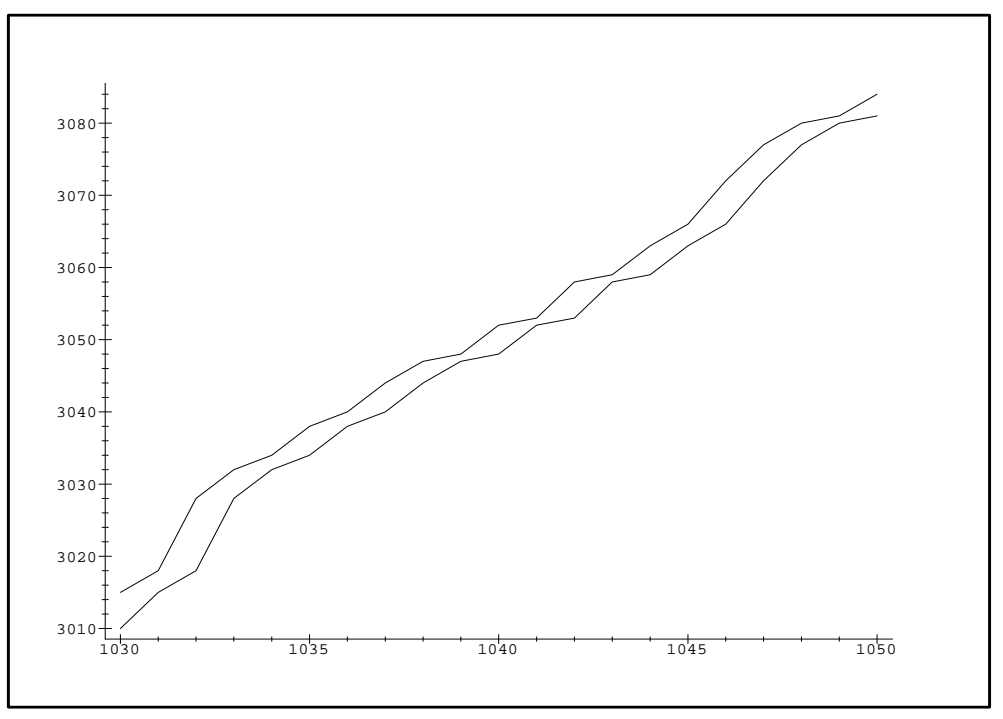

Fig. 9: Unnormalized $X(\cdot)$ with thickness (zoom). 
- $\operatorname{Pr}_{\ell}\left[T_{D} \geq x\right] \sim e^{-x / E_{\ell}\left[T_{D}\right]}, x \rightarrow \infty$.

We should write $C_{3}(\ell)$ but we will soon check that $C_{3}$ is independent of $\ell$. To compute $C_{3}$ and $\psi(\ell)$, we use the classical relation:

$$
E_{\ell}\left[T_{D}\right]=1+\sum_{j \in D^{C}} \Pi[\ell, j] E_{j}\left[T_{D}\right]
$$

i.e.

$$
\begin{aligned}
C_{3}+\psi(\ell) \varepsilon & =\varepsilon+\sum_{j \in D^{C}} \Pi[\ell, j]\left[C_{3}+\psi(j) \varepsilon\right]+O\left(\varepsilon^{2}\right) \\
& =\varepsilon+\Pi C_{3}-\varepsilon \varphi_{3}(\ell) C_{3}+\sum_{j} \Pi(\ell, j) \psi(j) \varepsilon+O\left(\varepsilon^{2}\right),
\end{aligned}
$$

and $\varphi_{3}$ is given by (II).

Eq. (14) leads to

$$
1=\sum_{D} \pi_{2}(\ell) E_{\ell}\left(T_{D}\right)
$$

(This is equivalent to a formula of Kac, see [15]).

Therefore, we obtain

$$
\begin{gathered}
1=C_{3} \sum_{D} \pi_{2}^{(1)}(\ell) \\
0=\sum_{D} \pi_{2}^{(1)}(\ell) \psi(\ell)+C_{3} \sum_{D} \pi_{2}^{(2)}(\ell)
\end{gathered}
$$

$\left(\pi_{2}^{(1)}\right.$ and $\pi_{2}^{(2)}$ are given by $\left.(9)\right)$.

Comparison of powers of $\varepsilon$ in (15) leads to $[I-\Pi] C_{3}=0$, which confirms that $C_{3}$ is independent of $\ell$. We derive

$$
\psi(\ell)=1-\varphi_{3}(\ell) C_{3}+\sum_{j} \Pi(\ell, j) \psi(j)
$$

or

$$
[I-\Pi] \psi=\delta, \quad \text { with } \quad \delta(\ell):=1-\varphi_{3}(\ell) C_{3} .
$$

We must have $\pi_{2} \delta=0$, therefore

$$
1=\sum \pi_{2}(\ell) \varphi_{3}(\ell) C_{3}, \quad C_{3}=1 / \sum_{\ell} \pi_{2}(\ell) \varphi_{3}(\ell)
$$

which fixes $C_{3}=-h_{w}\left(1-z^{*}\right)$. (This is of course equivalent to (16).)

We denote by $M_{1}$ the Drazin inverse of $I-\Pi$. We refer to Campbell and Meyer [5] for a detailed definition and analysis of the Drazin inverse. We have

$$
M_{1}=\sum_{n \geq 0}\left(\Pi^{n}-1 \times \pi_{2}\right)=M_{2}-1 \times \pi_{2}
$$

where $M_{2}:=\left[I-\Pi+1 \times \pi_{2}\right]^{-1}=\sum_{n \geq 0}\left[\Pi-1 \times \pi_{2}\right]^{n}$ is the potential used in Kemeny, Snell and Knapp [17]. The solution of (18) is given by $\psi=M_{1} \delta+\pi_{2} \psi$. 
To fix $C_{6}:=\pi_{2} \psi$, we first derive

$$
\sum_{D} \pi_{2}^{(1)} \psi=\sum_{D} \pi_{2}^{(1)} M_{1} \delta+C_{6} \sum_{D} \pi_{2}^{(1)}
$$

and with (177),

$$
C_{6}=\left[h_{w}\left(1-z^{*}\right) \frac{2}{h_{w}\left(1-z^{* 2}\right)}-\sum_{D} \pi_{2}^{(1)} M_{1} \delta\right]\left[-h_{w}\left(1-z^{*}\right)\right] .
$$

We can summarize our results in the following form.

Theorem 3.1 The thickness hitting time $E_{\ell}\left[T_{D}\right]$ to $D:=[k . . \infty], k>>1$ is given by

$$
E_{\ell}\left[T_{D}\right] \sim \frac{C_{3}}{z^{* k}}+\psi(\ell)+O\left(z^{* k}\right)
$$

with $C_{3}=-h_{w}\left(1-z^{*}\right), \psi(\ell)=M_{1}\left[1-C_{3} \varphi_{3}(\ell)\right]+C_{6}\left(M_{1}\right.$ is the potential kernel given in (19)).

$$
\operatorname{Pr}_{\ell}\left[T_{D} \geq x\right] \sim e^{-x z^{* k} / C_{3}}, x \rightarrow \infty
$$

Now let $\mathcal{M}(t):=\sup _{i \in[1 . . t]} x_{i}$ (maximum thickness with $t$ parts). We know that

$$
\operatorname{Pr}[\mathcal{M}(t)<k]=\operatorname{Pr}\left[T_{D} \geq t+1\right]
$$

So, by standard asymptotic analysis,

$$
\operatorname{Pr}[\mathcal{M}(t)<k] \sim \exp \left[-\exp \left[\log t-k \log \left(z^{*-1}\right)-\log C_{3}\right]\right], \quad t \rightarrow \infty .
$$

Set now $k:=j+1$ and $C_{4}=z^{*} / C_{3}$.

By Theorem 2.1, we know that $t=n \mu_{1}+O(\sqrt{n})$ and $\mu_{1}=h_{w} /\left(h_{z} z^{*}\right)$.

Hence, we derive the following theorem (we use now the notation $\mathcal{M}(n)$ ).

Theorem 3.2 Let

$$
\eta:=j-\left[\log n+\log C_{4}+\log \mu_{1}\right] / \log \left(z^{*-1}\right) .
$$

Then, with integer $j$ and $\eta=O(1)$,

$$
\operatorname{Pr}[\mathcal{M}(n) \leq j] \sim G_{1}(\eta), \quad n \rightarrow \infty
$$

where $G_{1}(\eta):=\exp \left[-\exp \left[-\eta \log \left(z^{*-1}\right)\right]\right]$. Let

$$
\psi_{1}(n):=\log \left(C_{4} n \mu_{1}\right) / \log \left(z^{*-1}\right)=\left[\log n-\log \left(-h_{z}\right)-\log \left(1-z^{*}\right)\right] / \log \left(z^{*-1}\right),
$$

and $\eta=j-\left\lfloor\psi_{1}(n)\right\rfloor-\left\{\psi_{1}(n)\right\}$. Asymptotically, the distribution is a periodic function of $\psi_{1}(n)($ with period 1), which can be written as

$$
\log \operatorname{Pr}\left[\mathcal{M}(n) \leq\left(\left\lfloor\psi_{1}(n)\right\rfloor+k\right) e^{-\left\{\psi_{1}(n)\right\} \log \left(z^{*-1}\right)} \underset{n \rightarrow \infty}{\longrightarrow}-e^{-k \log \left(z^{*-1}\right)} .\right.
$$

We also have

$$
\operatorname{Pr}[\mathcal{M}(n)=j] \sim f_{1}(\eta)
$$

where $f_{1}(\eta):=G_{1}(\eta)-G_{1}(\eta-1)$. 


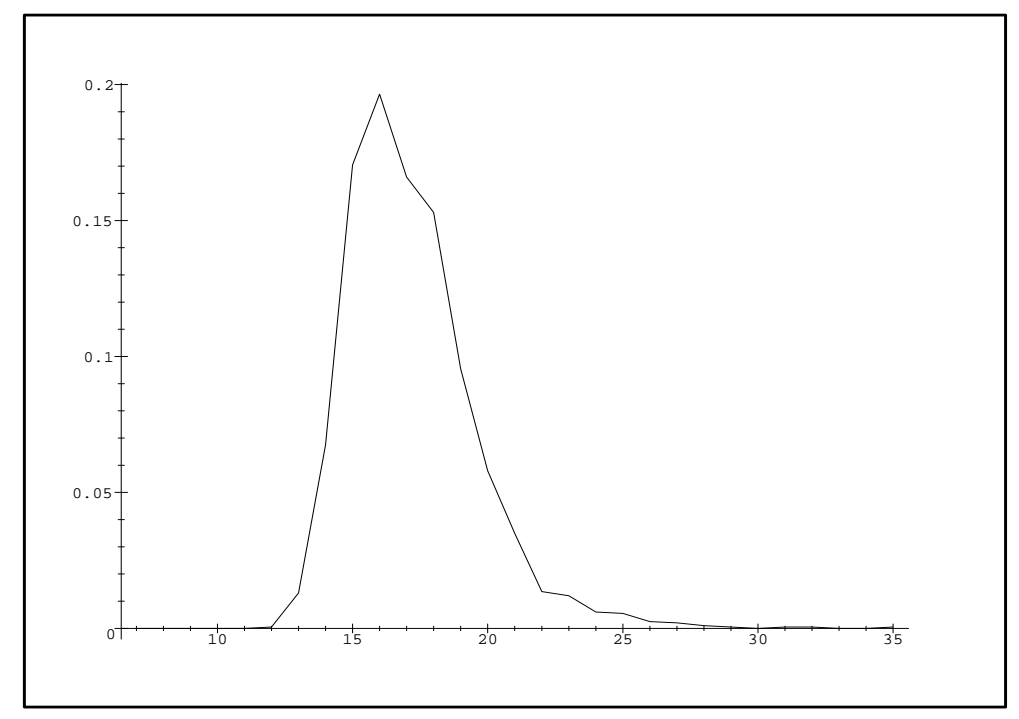

Fig. 10: Distribution of maximum thickness.

The asymptotic moments of $\mathcal{M}(n)$ are also periodic functions of $\psi_{1}(n)$. They can be written as Harmonic sums which are usually analyzed with Mellin transforms: see Flajolet et al [ []$]$. The asymptotic non-periodic term in the moments of $\mathcal{M}(n)$ is given by the following result.

Theorem 3.3 The constant term $\bar{E}$ in the Fourier expansion (in $\psi_{1}(n)$ ) of the moments of $\mathcal{M}(n)$ is asymptotically given by

$$
\bar{E}\left[\mathcal{M}(n)-\psi_{1}(n)\right]^{i} \sim \int_{-\infty}^{+\infty} \eta^{i}\left[G_{1}(\eta)-G_{1}(\eta-1)\right] d \eta
$$

It is well known that the extreme-value distribution function $e^{-e^{-x}}$ has mean $\gamma$ and variance $\pi^{2} / 6$. From this, we can for instance derive

$$
\bar{E}[\mathcal{M}(n)] \sim \psi_{1}(n)+\frac{1}{2}+\frac{\gamma}{\log \left(z^{*-1}\right)} .
$$

The other periodic terms have very small amplitude (see Flajolet et al [7]]).

For $n=10.000$, we have simulated 2.000 trajectories. The observed distribution for maximum thickness is given in Figure 10.

The observed and limiting distribution function (DF) are given in Figure $[1]$ (observed = circle, asymptotic $=$ line). The limiting DF is given by $G_{1}\left[j-\psi_{1}(n)\right]$ and, due to the sensitivity of $G_{1}$ to the mean, we have taken $\psi_{1}(n)=\overline{\mathcal{M}}_{0}(n)-\frac{1}{2}-\frac{\gamma}{\log \left(z^{*-1}\right)}$ where $\overline{\mathcal{M}}_{0}(n)$ is the observed mean $=17 \cdot 17 \ldots$ The limiting mean $\bar{E}(\mathcal{M}(n))$ is given by (20) and leads to $17 \cdot 09739171 \ldots$ 


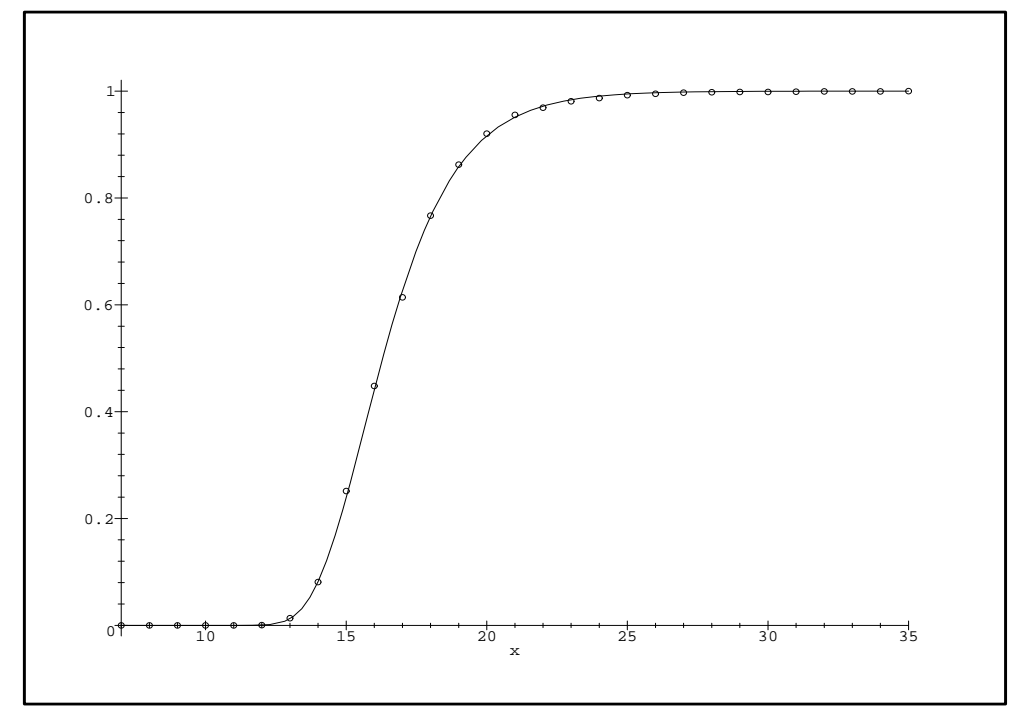

Fig. 11: Observed and limiting DF.

\section{Conclusion}

Using some generating functions and limit convergence theorems, we have obtained a complete stochastic description of Carlitz compositions. The same techniques could be used for some generalizations such as Carlitz compositions with zeros and with some restrictions. In a recent paper by Hitczenko and Louchard [12], the distinctness of classical and Carlitz compositions has been fully analyzed.

\section{Acknowledgements}

The pertinent comments of the referee led to improvements in the presentation.

\section{References}

[1] D. Aldous. Probability Approximations via the Poisson Clumping Heuristic. Springer-Verlag, 1989.

[2] E.A. Bender. Central and local limit theorems applied to asymptotics enumeration. Journal of Combinatorial Theory, Series A, 15:91-111, 1973.

[3] Edward A. Bender. Convex n-ominoes. Discrete Math., 8:219-226, 1974.

[4] P. Billingsley. Convergence of Probability Measures. Wiley, 1968.

[5] S. L. Campbell and C. D. Meyer. Generalized Inverse of Linear Transformations. Pitman, 1979. 
[6] L. Carlitz. Restricted compositions. Fibonacci Quarterly, 14:254-264, 1976.

[7] P. Flajolet, X. Gourdon, and P. Dumas. Mellin transforms and asymptotics: Harmonic sums. Theoretical Computer Science, 144:3-58, 1995.

[8] P. Flajolet and H. Prodinger. Level number sequences for trees. Discrete Mathematics, 65:149-156, 1987.

[9] P. Flajolet and M. Soria. General combinatorial schemes: Gaussian limit distribution and exponential tails. Discrete Mathematics, 114:159-180, 1993.

[10] I. P. Goulden and D. M. Jackson. Combinatorial enumeration. John Wiley \& Sons Inc., New York, 1983. With a foreword by Gian-Carlo Rota, Wiley-Interscience Series in Discrete Mathematics.

[11] P. Henrici. Applied and Computational Complex Analysis. Wiley, 1988.

[12] P. Hitczenko and G. Louchard. Distinctness of compositions of an integer: a probabilistic analysis. Random Structures and Algorithms, 19(3,4):407-437, 2001.

[13] H.K. Hwang. Théorèmes limites pour les structures aléatoires et les fonctions arithmétiques. 1994. Thèse, Ecole Polytechnique de Palaiseau.

[14] H.K. Hwang. On convergence rates in the central limit theorems for combinatorial structures. European Journal of Combinatorics, 19:329-343, 1998.

[15] M. Kac. On the notion of recurrence in discrete stochastic processes. Bulletin of the American Mathematical Society, 53:1002-1019.

[16] J. Keilson. Markov Chain Models-Rarity and Exponentiality. Springer-Verlag, 1979.

[17] J. G. Kemeny, J. L. Snell, and A. W. Knapp. Denumerable Markov Chains. Van Nostrand, 1966.

[18] A. Knopfmacher and H. Prodinger. On Carlitz compositions. European Journal of Combinatorics, 19:579-589, 1998.

[19] G. Louchard. Probabilistic analysis of some (un)directed animals. Theoretical Computer Science, 159(1):65-79, 1996.

[20] G. Louchard. Probabilistic analysis of column-convex and directed diagonally-convex animals. Random Structures and Algorithms, 11:151-178, 1997.

[21] G. Louchard. Probabilistic analysis of column-convex and directed diagonally-convex animals. II: Trajectories and shapes. Random Structures and Algorithms, 15:1-23, 1999.

[22] E.T. Whittaker and G.N. Watson. A Course of Modern Analysis. Cambridge University press (reprinted 1973), 1927. 


\section{A Appendix : Some combinatorial identities}

Let us first set $T(a, b, c):=\sum_{k \geq 1} k^{a} \frac{z^{b k}}{\left(1+z^{k}\right)^{c}}$.

$$
\sum_{1}^{\infty} \frac{z^{* j}}{1+z^{* j}}=1
$$

Proof

$$
\begin{gathered}
\sum_{j \geq 1}(-1)^{j+1} \frac{z^{j}}{1-z^{j}}=\sum_{j \geq 1}(-1)^{j+1} \sum_{k \geq 1} z^{k j} \\
=-\sum_{k, j \geq 1}\left(-z^{k}\right)^{j}=T(0,1,1)
\end{gathered}
$$

Since for the special choice $z^{*}$ of $z$ the left side equals 1 , the right side also does. Note that this is the only identity where we need the value $z^{*}$. All next identities are on arbitrary $z$.

$$
\sum_{1}^{\infty} \frac{z^{* j}}{\left(1+z^{* j}\right)^{2}}=-h_{w}
$$

Proof

$$
h(w, z)=1+\sum_{j \geq 1}(-1)^{j} \frac{z^{j} w^{j}}{1-z^{j}} .
$$

Hence

$$
\begin{aligned}
-h_{w} & =\sum_{j \geq 1}(-1)^{j+1} \frac{j z^{j}}{1-z^{j}} \\
& =\sum_{j, k \geq 1}(-1)^{j+1} j z^{j k} \\
& =-\sum_{j, k \geq 1} j\left(-z^{k}\right)^{j} \\
& =T(0,1,2) .
\end{aligned}
$$

$$
\sum_{1}^{\infty} \frac{j z^{* j}}{\left(1+z^{* j}\right)^{2}}=-z^{*} h_{z} .
$$

Proof

$$
-z h_{z}=\sum_{j \geq 1}(-1)^{j+1} \frac{j z^{j}}{\left(1-z^{j}\right)^{2}}
$$




$$
\begin{aligned}
& =\sum_{j, k \geq 1}(-1)^{j+1} j k z^{j k} \\
& =-\sum_{j, k \geq 1} k j\left(-z^{k}\right)^{j} \\
& =T(1,1,2) .
\end{aligned}
$$

$$
\sigma_{2}^{2} \equiv \sigma_{X}^{2} . \quad(\mathrm{A} 4)
$$

Proof

$$
\begin{gathered}
S_{2}=\frac{T(2,1,2)}{T(0,1,2)}, \\
\mu_{2}^{2}=\left(\frac{z h_{z}}{h_{w}}\right)^{2}=\left(\frac{T(1,1,2)}{T(0,1,2)}\right)^{2}, \\
\bar{\varphi}_{6}=-\frac{T(2,2,3)}{T(0,1,2)}, \\
\sigma_{X}^{2}=S_{2}-\mu_{2}^{2}+2 \Sigma,
\end{gathered}
$$

or, by (113),

$$
\begin{aligned}
\frac{T(2,1,2)}{T(0,1,2)}+\left(\frac{T(1,1,2)}{T(0,1,2)}\right)^{2} & -2 \frac{T(2,2,3)}{T(0,1,2)}-2 \frac{\bar{\varphi}_{5, w}}{T(0,1,2)^{2}}-\frac{\bar{\varphi}_{5} h_{w w}}{(T(0,1,2))^{3}} . \\
h_{w w} & =\sum_{j \geq 1}(-1)^{j+1} \frac{j(j-1) z^{j}}{1-z^{j}} \\
& =\sum_{j, k \geq 1}(-1)^{j+1} j(j-1) z^{j k} \\
& =-\sum_{j, k \geq 1} j(j-1)\left(-z^{k}\right)^{j} \\
& =2 T(0,2,3) . \\
\bar{\varphi}_{5}= & h_{w}^{2} \mu_{2}^{2}=\left(z h_{z}\right)^{2}=T(1,1,2)^{2} . \\
\varphi_{5}(w, \theta, i) & =\sum_{k \geq 1}(-1)^{k+1} \frac{z^{k} \theta w^{k}}{1-\theta z^{k}} \cdot \frac{z^{i}}{1+w z^{i}} .
\end{aligned}
$$




$$
\begin{gathered}
{\left[\theta^{j}\right] \varphi_{5}(w, \theta, i)=\sum_{k \geq 1}(-1)^{k+1} z^{k} w^{k} z^{k(j-1)} \frac{z^{i}}{1+w z^{i}}} \\
=\sum_{k \geq 1}(-1)^{k+1} z^{k j} w^{k} z^{i} \sum_{\ell \geq 0}(-1)^{\ell}\left(w z^{i}\right)^{\ell} . \\
{\left[\theta^{j}\right] \varphi_{5, w}(1, \theta, i)=\sum_{\ell \geq 0} \sum_{k \geq 1}(-1)^{k+1} z^{k j} z^{i} z^{i \ell}(k+\ell)(-1)^{\ell} .} \\
\bar{\varphi}_{5, w}(1)=\sum_{i \geq 1} \sum_{j \geq 1} \sum_{\ell \geq 0} \sum_{k \geq 1} \frac{i}{1+z^{i}} \frac{j}{1+z^{j}}(-1)^{k+1} z^{k j} z^{i} z^{i \ell}(k+\ell)(-1)^{\ell} \\
=-\sum_{i \geq 1} \frac{i z^{2 i}}{\left(1+z^{i}\right)^{3}} \sum_{j \geq 1} \frac{j z^{j}}{\left(1+z^{j}\right)^{2}}+\sum_{i \geq 1} \frac{i z^{i}}{\left(1+z^{i}\right)^{2}} \sum_{j \geq 1} \frac{j z^{j}}{\left(1+z^{j}\right)^{3}} .
\end{gathered}
$$

This gives

$$
\bar{\varphi}_{5, w}(1)=T(1,1,2)[T(1,1,3)-T(1,2,3)] .
$$

So $\sigma_{X}^{2}$ becomes

$$
\begin{aligned}
\sigma_{X}^{2} & =\frac{T(2,1,2)}{T(0,1,2)}+\frac{T(1,1,2)^{2}}{T(0,1,2)^{2}}-2 \frac{T(2,2,3)}{T(0,1,2)} \\
& -2 \frac{T(1,1,2)[T(1,1,3)-T(1,2,3)]}{T(0,1,2)^{2}}-2 \frac{T(1,1,2)^{2} T(0,2,3)}{T(0,1,2)^{3}}
\end{aligned}
$$

This should equal $\sigma_{2}^{2}=\sigma_{1}^{2} / \mu_{1}^{3}=1 / \mu_{1}-\frac{r_{2}}{3 \mu_{1}^{3}}$.

But, after a boring but simple computation we find

$$
\begin{gathered}
h_{z z}=\frac{1}{z^{2}}[T(2,2,3)+T(1,2,3)-T(2,1,3)+T(1,1,3)], \\
\mu_{1}=\frac{h_{w}}{z h_{z}}=\frac{T(0,1,2)}{T(1,1,2)}, \\
h_{w w}=2 T(0,2,3), \\
h_{z w}=\frac{1}{z}[T(1,2,3)-T(1,1,3)] .
\end{gathered}
$$

Therefore 


$$
\begin{aligned}
& \sigma_{2}^{2}=\frac{\sigma_{1}^{2}}{\mu_{1}^{3}}=\frac{1}{\mu_{1}}-\frac{r_{2}}{z \mu_{1}^{3}} \\
& =\frac{T(1,1,2)}{T(0,1,2)}-\frac{r_{2}}{z} \frac{T(1,1,2)^{3}}{T(0,1,2)^{3}} \\
& =\frac{T(1,1,2)}{T(0,1,2)}+\frac{r_{1}^{2} h_{z z}+2 r_{1} h_{z w}+h_{w}+h_{w w}}{z h_{z}} \frac{T(1,1,2)^{3}}{T(0,1,2)^{3}} \\
& =\frac{T(1,1,2)}{T(0,1,2)}-\frac{r_{1}^{2} h_{z z}+2 r_{1} h_{z w}+h_{w}+h_{w w}}{T(1,1,2)} \frac{T(1,1,2)^{3}}{T(0,1,2)^{3}} \\
& =\frac{T(1,1,2)}{T(0,1,2)}-\left[r_{1}^{2} h_{z z}+2 r_{1} h_{z w}+h_{w}+h_{w w}\right] \frac{T(1,1,2)^{2}}{T(0,1,2)^{3}} \\
& =\frac{T(1,1,2)}{T(0,1,2)}-\frac{T(0,1,2)^{2}}{T(1,1,2)^{2}}[T(2,2,3)+T(1,2,3)-T(2,1,3)+T(1,1,3)] \frac{T(1,1,2)^{2}}{T(0,1,2)^{3}} \\
& -\left[2 r_{1} h_{z w}+h_{w}+h_{w w}\right] \frac{T(1,1,2)^{2}}{T(0,1,2)^{3}} \\
& =\frac{T(1,1,2)}{T(0,1,2)}-[T(2,2,3)+T(1,2,3)-T(2,1,3)+T(1,1,3)] \frac{1}{T(0,1,2)} \\
& -\left[2 r_{1} h_{z w}+h_{w}+h_{w w}\right] \frac{T(1,1,2)^{2}}{T(0,1,2)^{3}} \\
& =\frac{T(1,1,2)}{T(0,1,2)}-\frac{T(2,2,3)}{T(0,1,2)}-\frac{T(1,2,3)}{T(0,1,2)}+\frac{T(2,1,3)}{T(0,1,2)}-\frac{T(1,1,3)}{T(0,1,2)} \\
& -\left[2 r_{1} h_{z w}+h_{w}+h_{w w}\right] \frac{T(1,1,2)^{2}}{T(0,1,2)^{3}} \\
& =\frac{T(1,1,2)}{T(0,1,2)}-\frac{T(2,2,3)}{T(0,1,2)}-\frac{T(1,2,3)}{T(0,1,2)}+\frac{T(2,1,3)}{T(0,1,2)}-\frac{T(1,1,3)}{T(0,1,2)} \\
& +2 \frac{T(0,1,2)}{T(1,1,2)}[T(1,2,3)-T(1,1,3)] \frac{T(1,1,2)^{2}}{T(0,1,2)^{3}} \\
& -\left[h_{w}+h_{w w}\right] \frac{T(1,1,2)^{2}}{T(0,1,2)^{3}} \\
& =\frac{T(1,1,2)}{T(0,1,2)}-\frac{T(2,2,3)}{T(0,1,2)}-\frac{T(1,2,3)}{T(0,1,2)}+\frac{T(2,1,3)}{T(0,1,2)}-\frac{T(1,1,3)}{T(0,1,2)} \\
& +2[T(1,2,3)-T(1,1,3)] \frac{T(1,1,2)}{T(0,1,2)^{2}} \\
& -[-T(0,1,2)+2 T(0,2,3)] \frac{T(1,1,2)^{2}}{T(0,1,2)^{3}} \\
& =\frac{T(1,1,2)}{T(0,1,2)}-\frac{T(2,2,3)}{T(0,1,2)}-\frac{T(1,2,3)}{T(0,1,2)}+\frac{T(2,1,3)}{T(0,1,2)}-\frac{T(1,1,3)}{T(0,1,2)}
\end{aligned}
$$




$$
\begin{aligned}
& +\quad 2[T(1,2,3)-T(1,1,3)] \frac{T(1,1,2)}{T(0,1,2)^{2}} \\
& +\quad \frac{T(1,1,2)^{2}}{T(0,1,2)^{2}}-2 T(0,2,3) \frac{T(1,1,2)^{2}}{T(0,1,2)^{3}}
\end{aligned}
$$

But now, we easily check that

$$
T(2,1,3)-T(2,2,3) \equiv-2 T(2,2,3)+T(2,1,2),
$$

and

$$
T(1,1,3)+T(1,2,3)=T(1,1,2) .
$$

Identification of $\sigma_{2}^{2}$ and $\sigma_{X}^{2}$ is now immediate. 
